\title{
Representing energy. I. Representing a substance ontology for energy
}

\author{
Rachel E. Scherr, ${ }^{1}$ Hunter G. Close, ${ }^{2}$ Sarah B. McKagan, ${ }^{1}$ and Stamatis Vokos ${ }^{1}$ \\ ${ }^{1}$ Department of Physics, Seattle Pacific University, Seattle, Washington 98119, USA \\ ${ }^{2}$ Department of Physics, Texas State University, San Marcos, Texas 78666, USA
}

(Received 17 April 2012; published 19 October 2012)

\begin{abstract}
The nature of energy is not typically an explicit topic of physics instruction. Nonetheless, verbal and graphical representations of energy articulate models in which energy is conceptualized as a quasimaterial substance, a stimulus, or a vertical location. We argue that a substance ontology for energy is particularly productive in developing understanding of energy transfers and transformations. We analyze classic representations of energy — bar charts, pie charts, and others - to determine the energy ontologies that are implicit in those representations, and thus their affordances for energy learning. We find that while existing representations partially support a substance ontology for energy and thus the learning goal of energy conservation, they have limited utility for tracking the flow of energy among objects.
\end{abstract}

DOI: 10.1103/PhysRevSTPER.8.020114

PACS numbers: 01.40.Fk, 01.40.Ha, 01.40.J-

\section{INTRODUCTION}

Representations are at the heart of the scientific enterprise. Central practices of science include many representation-based activities, including representational invention, refinement, negotiation, adaptation, adoption, rule-based manipulation, and enrichment or pruning of features of representations. The Newtonian synthesis, for instance, was accomplished through the representational tool of forces, which also included the mathematization of interactions between distant objects-yet another layer of representation. The imputed stresses and strains in the luminiferous aether gave rise, in Maxwell's equations, to quantitative representations of the dynamics of invented quantities, namely, of electric and magnetic fields. Lewis structures represent the ordered nature of attractive interactions between elements, the arrangement of which according to certain rules gives rise to yet another powerful representation, the periodic table of elements. Feynman diagrams represent in a graphical sense the workings of perturbative quantum field theory, which itself is a representation of certain types of interactions. Our current understanding of fundamental interactions is based on group theory representations, which themselves represent symmetry in a codified way. Genomic research is based on a certain letter-based grammar, itself a powerful representation. Science itself is a representation of a class of patterns identified and manipulated by the community of its practitioners. This vision is consistent with the longstanding practice of using student skill with the use of sanctioned representations as a proxy for understanding the conceptual structure of the relevant domain. For

Published by the American Physical Society under the terms of the Creative Commons Attribution 3.0 License. Further distribution of this work must maintain attribution to the author(s) and the published article's title, journal citation, and DOI. instance, free-body diagrams play a major role in assessing conceptual understanding of Newtonian dynamics; kinematics graphs play a similarly large role in evaluating whether or not students understand principles of motion; potential energy graphs are omnipresent in quantum mechanics textbooks.

A representation is not only a tool for making sense of the scientifically adopted canon, but also an activity for creating scientific knowledge. Following philosopher van Fraassen in Ref. [1] we claim that representation can mean both "a product" and "an activity" — both a visual or tangible depiction of a scientific phenomenon and the process of creating that depiction. Though our focus in this paper is on the product more than the coconstructive process, we see the features of a representation as inextricably related to the community of participants that generated it, sustain its meaning, and are, at least in principle, aware of its affordances and limitations.

We view scientific representations as being culturally produced cognitive agents that speak with the voice of the scientific culture that produced them [2-5]. Our perspective contrasts with a Baconian approach to science, in which data speak for themselves. Our particular interest is in representations of energy, one of the most famously abstract quantities in introductory physics. A key part of what an energy representation articulates is what kind of thing energy is (its ontology). The nature of energy is not typically an explicit topic of physics instruction. Nonetheless, the spoken and written discourse of both physics learners and disciplinary experts suggests conceptualizations of energy (Sec. II). We identify three main ontologies: one in which energy is understood as a metaphorical substance, one in which it is seen as a type of stimulus, and one in which it is symbolized by vertical locations. We argue that a substance metaphor for energy is valuable for specific learning goals, including energy conservation and tracking the transfers and transformations of 
energy within, into, or out of systems of objects (Secs. III and IV). Finally, we analyze a variety of classic representations, such as bar charts and pie charts, to determine their affordances for energy learning (Sec. V). Guiding questions include

- What do specific representations "say" about energy?

- What questions do specific representations "ask" of one who engages with them?

- Which representations best support our learning goals?

We find that while each of the aforementioned representations partially supports a substance metaphor for energy, they have limited utility for tracking the flow of energy among objects. In a companion paper [5], we present a novel representation that embodies the substance metaphor and supports learners in conserving and tracking energy as it flows from object to object and changes form. Such representations enable detailed modeling of energy dynamics in complex physical processes.

\section{ONTOLOGIES FOR ENERGY IN LEARNER AND EXPERT DISCOURSE}

An embodied cognition theoretical framework structures our thinking about how people conceptualize abstractions in general. In this section we describe the ontologies that we have found to structure thinking about energy in physics discourse, and discuss the advantages and limitations of the substance ontology, which is a critical piece of our instructional approach. (Some of the material in this section appeared in Ref. [6].)

In an embodied cognition perspective, all abstractions are understood in terms of basic sensory-motor experiences such as object permanence and movement $[7,8]$. Ideas such as time are expressed with embodied metaphors: for example, we might say that we are "halfway through" the year, as though a year had spatial extent and we were moving relative to it. Human use of embodied metaphors is natural, unconscious, and pervades our talk; we often express conceptualizations of events, activities, emotions, ideas, and so on as being entities or substances. Embodied metaphors are often especially evident in the verbs and prepositional phrases used together with the terms of interest. For example, to say someone is in trouble or close to graduation conceptualizes these states as being locations, and to say that someone got an idea or has a headache poses these attributes as being possessions [7]. We use grammatical indicators such as these to identify learner ontologies for energy - the kinds of things that people think of energy as being. Influential research in cognitive science has demonstrated that ontological categorization is key to understanding physics concepts [9-11].

We identify three main ontologies for energy in learner and expert physics discourse: (1) a quasimaterial substance, (2) a stimulus to action, and (3) a vertical location. The ontologies that we identify are consistent with conceptualizations of energy identified in both younger and older children [12-16]. Those earlier studies, however, emphasize the diversity and variability of student ideas, which are selectively evoked depending on the research method used and energy scenarios presented. We share with Amin [17] an interest in identifying a few key metaphors signifying learner understandings of the nature of energy.

We illustrate our analysis methodology and results with excerpts from video records of an eighth-grade classroom in a public middle school in the Pacific Northwest and from physics textbooks. The video data were collected as part of a professional development activity in which participating teachers had their classrooms videotaped for discussion with peer teachers and researchers. The episode below was selected because of the high visibility of students' science ideas. The correctness or incorrectness of their ideas was not part of the selection process. Students in the episode have just begun their study of energy by watching a movie showing various phenomena: a bus driving, a bicyclist pedaling, leaves blowing in the street, and so on. After the movie, students work in small groups on a worksheet that asks them to decide how energy is involved in each of the phenomena in the movie. Five students participate in the following discussion:

Danielle: All right. Leaves in street. I don't think so. Cause it's just the wind.

Brianna: Yeah. I don't think I don't think the leaves in the street have energy. They have a type of energy, but-

Emily: But is wind energy?

Brianna: Wind is energy.

Danielle: Oh, should we write it down?

Emily: Well they're getting wind energy.

Christopher: But it's not talking about the wind.

Brianna: -about the wind, it's just talking about the leaves in the street. Leaves-leaves in the street, do they have energy.

Danielle: No.

Christopher: They are pushed by energy

Brianna: They have energy, but they do not have the energy, like, to move.

Brianna: But wind-I don't think wind has energy. Well it has energy

Emily: You can use wind to like power stuff

Brianna: Is it-to create energy. Wind-

Emily: It can be a source of energy.

Brianna: Yeah, your wind is like your foot on the pedals.

Emily: Yeah. It's a source of energy.

Brianna: I don't really think-

Emily: I mean the leaves have a source of energy.

Brianna: The leaves have a source of energy but they don't have like energy to move they're talking about its motion. Is motion energy there and I don't think the leaves have motion energy. 
$\ldots$

Emily: But the leaves were moving.

Brianna: But they're moving because of the wind. I'm saying if you could have-

Emily: That is their source of energy.

Brianna: -leaves in the street without, without, if we had leaves without the wind, it wouldn't move then. I'm just thinking of both cases.

Emily: True but I mean but like a bus without gasoline wouldn't move either.

Brianna: No, true.

Emily: And like a bicycle without pedals, a pedaler, wouldn't move either. So.

Brianna: I don't know I just feel like a leaf wouldn't

Ashley: It seems like it is but

Brianna: But I feel like in my mind I feel like it's energy! It's involved in a type of energy. I'm going to put yesslash-no, because it's involved in a type of energy.

In what follows, we identify three different ontologies for energy that are implicit in the above conversation and in additional material from physics textbooks. We infer these ontologies on the basis of grammatical indicators, especially verbs and prepositional phrases $[9,18,19]$.

\section{A. Substance}

Certain statements pose energy as being a substancelike quantity - a kind of "stuff" — and objects as being containers that can have such stuff in them:

Leaves blowing in the street have energy.

They're getting wind energy.

Statements that implicitly treat energy as a substance are ubiquitous in physics textbooks and the words of famous physicists. Even statements that carefully avoid any explicit characterization of energy as anything other than an abstract numerical quantity use an implicit substance metaphor:

We now introduce a third type of energy that a system can possess [20].

Thus, the flying duck has a kinetic energy of $6.0 \mathrm{~J}$ [21].

... when we put energy into the gas its molecules move faster and so the gas gets heavier [22]. ${ }^{1}$

This imagined substance is not a material fluid; rather it is a "quasimaterial" substance, one that includes certain properties of material substances (e.g., localization and conservation) but not others (e.g., mass, volume, viscosity). As we will discuss in more depth below, the substance metaphor for energy is a powerful conceptualization that supports features valued in physics, specifically conservation, presence in objects, transfer, and flow [17,19,23-25].

In a teacher professional development course, participants offered a refinement of the substance metaphor in

\footnotetext{
${ }^{1}$ See Ref. [17] for more examples of the manner in which Feynman speaks metaphorically about energy.
}

which energy is infused into objects the way tea flavor is infused into water. This image retains the sense of energy as a kind of stuff that can be in objects, but is distinctive in that energy is understood as permeating solid objects and changing their quality without adding significant mass or volume, similar to an expert model of electric charge.

Some other statements characterize energy as being something like fuel, and of certain kinds of objects as possessing fuel energy:

Leaves wouldn't move without wind as a bus wouldn't move without gasoline.

Wind can be a source of energy.

You can use wind to power stuff.

Fuel is not energy; rather, it is a (literal) material substance that contains energy and (taken together with oxygen) can transfer that energy to other objects at a selected time. In physics, any object can possess and transfer energy. Fuel is distinctive in that the energy of interest is often chemical energy; the transfer often takes place by combustion; and the desired effect of the energy transfer is to result in mechanical work, so that the energy of interest is the "useful" energy and the objects of interest are those we use as "power sources" (wind, gasoline, batteries, food). The fuel metaphor is also apparent when expert physicists talk about energy being used, stored, and extracted:

We spend a tremendous amount of money to acquire and use energy [21].

... the elastic potential energy stored in the spring is $\frac{1}{2} k x^{2}$ [20].

... all we would have done would be to extract energy from the reservoir at $\mathrm{T} 2$ ! [22].

Fuel is unlike energy in that it is used up (transformed into a nonfuel substance). Nonetheless, the strong everyday association between energy and fuel may be a resource for instruction if learners can be persuaded to think of fuel as having (and giving) energy rather than being energy. The burning of fuel may also support understanding the second law of thermodynamics in that it is a compelling everyday example of an irreversible process.

\section{B. Stimulus}

Other statements pose energy as being a stimulus, whose primary property is that it has an effect on objects. In some cases, this stimulus is spoken of as being much like what experts would term a force:

Leaves in the street are pushed by energy.

In other cases, energy is seen as being an agent that exerts something like a force:

Wind is energy.

Other statements characterize energy (or an energyassociated entity) as a general trigger or impetus for action:

Wind is like your foot on a pedal. 
Leaves wouldn't move without wind as a bicycle wouldn't move without $a$ pedaler.

In other contexts, we have observed energy being spoken of as a stimulus whose effects are specific to the object being stimulated: magnetizable things become magnets, stretchy things get stretched, lights turn on, movable things get moving. Such objects may be described as being "energized," in the sense of being roused.

The stimulus metaphor for energy is distinct from the substance metaphor in that instead of energy being in objects (as a fluid is in a container), energy acts on objects (as a stick prods a goat). The stimulus metaphor is a conceptualization that supports features valued in sociopolitical discourse, specifically the necessity of energy for sustaining activity. It also supports the idea that energy is the "ability to do work," and to some extent the causal mechanistic relationship between energy and forces. It is also problematic for this relationship in that it does not clearly differentiate energy from forces. Further, the stimulus metaphor does not support conservation: forces (or more general trigger or impetus mechanisms) can appear and disappear without constraint and do not transfer from one object to another. More generally, defining energy as the "ability (or capacity) to do work" does not promote conservation, as "ability" and "capacity" are not easily understood as conserved quantities. For this reason, we do not emphasize the stimulus metaphor or its associated vocabulary in instruction. Though the "ability" definition of energy is common, the stimulus metaphor is not common in expert physicists' discourse about energy.

\section{Vertical location}

A third ontology for energy is in place when we speak of energy as being an ordered set of vertical locations; for example, "The kinetic energy of the cart gets higher as the cart speeds up." (Both higher and up as used here indicate common orientational metaphors, in this case "more is up," [7] but only higher refers to energy). A graph with energy on the vertical axis is a diagrammatic representation using this same metaphor. In these examples, the set of vertical locations is implicitly understood to be continuous. In the study of quantum harmonic oscillators or atoms, discourse arises of discrete energy levels. This metaphor did not arise in the episode about the leaves in the street, probably because the discussion focused on a qualitative, rather than a quantitative, understanding of energy. Physics textbooks, however, commonly include statements such as the following:

The electron makes a transition from the $n=2$ energy level to the ground level $(n=1)$ [20].

One way an electron makes a quantum jump up to a greater energy level is to absorb a photon [21].

When we try to push the atoms very close together the energy goes up very rapidly, because they repel each other [22].
Most physics textbooks show a graphical version of the vertical location metaphor with energy-level diagrams, in which energies are visualized as rungs on a ladder and transitions are represented as vertical arrows. In these textual and graphic (implicit) statements of the nature of energy, energy is not in an object (substance metaphor), nor does it act on an object (stimulus metaphor); rather, objects are at energies (location metaphor).

The vertical location metaphor is a conceptualization that supports the idea that effort must be exerted to increase the energy of an object, consistent with the first law of thermodynamics. It harnesses many of the same cognitive resources as the concept of gravitational potential energy, which increases with (literal) height.

There are pitfalls if students take this metaphor literally when studying potential barriers, steps, and wells in quantum mechanics [26-28]. There are also advantages of this metaphor for students and for expert physicists. Relating the behavior of objects in potential wells and barriers to the behavior of balls on actual hills and valleys can help students understand these abstract concepts in terms of more familiar concepts [29]. Expert physicists use this metaphor to understand the complex behavior of energy levels and particles in response to various potential energy configurations; for example, thinking of decreasing the width of a potential well as "squeezing" energy levels and particles to make them rise [30]. Potential wells are two-dimensional representations, in which the vertical dimension of space symbolizes energy, but the horizontal dimension symbolizes configuration space; yet for expert physicists, it can be productive to think of the two dimensions as interacting with each other.

\section{USING THE SUBSTANCE ONTOLOGY IN INSTRUCTION}

Instructors who appreciate the advantages and limitations of various metaphors for physical quantities are better prepared to understand their significance in student discourse and use them as a resource for helping students deepen their understanding. The substance metaphor has attracted considerable attention from both physicists and researchers in the cognitive sciences. In each case, some argue for its advantages and others warn of attendant risks. Our position is that a substance metaphor for energy has advantages for physics understanding that outweigh the concerns.

\section{A. Advantages of a substance ontology for energy}

We have selected a substance metaphor for energy as a primary focus of our instruction because of its advantages for teaching conservation, transfer, and flow [23-25,31-33]. A substance metaphor supports the following features:

(i) Energy is conserved. This key feature is a primary advantage of the metaphor. 
(ii) Energy is localized, i.e., it is associated with a spatial location, even if spread out.

(iii) Energy is located in objects, which are metaphorically represented as containers for energy.

(iv) Energy can change form. Forms, in our model, are categories of evidence that energy is present or changing, and thus an important means of connecting a unified energy concept to a variety of observable phenomena [34].

(v) (a) Energy is transferred among objects and (b) energy can accumulate in objects. Flow corresponding to a conserved quantity (i.e., a quantity subject to the continuity equation) is a key concept in physics, appearing here as energy transfer (and elsewhere as mass transfer, charge transfer, and momentum transfer, in both classical and quantum mechanical contexts).

These features constitute a powerful conceptual model of energy that may be used to explain and predict energy phenomena.

Substance conceptualizations of physics concepts have been held responsible for a large number of physics misconceptions [35]. Such research specifically highlights force, heat, light, and electric current as processes (occurrences over an extended time interval) that novices miscategorize as substances (types of material) [9-11,19,35]. For example, if students incorrectly think of force as a substance, they might tend to think of it as residing in a single object. They might then be supported in the idea that a contact force persists in an object after the object is no longer in contact with the agent causing the force, and runs out as the object slows down. To our knowledge, the cited research does not address the ontology of energy; instead, the focus is on processes of energy transfer. However, since processes of energy transfer (e.g., heat) are identified as processes, we are led to presume that energy itself might be placed in the substance ontological category. If so, then a substance conceptualization of energy would be ontologically correct, and thus offer an important advantage for learning (including distinguishing energy from forces).

Conceptualizing energy as a substance is especially powerful-perhaps inevitable, and perhaps even cognitively necessary - for understanding energy as a conserved quantity. Planck attributes the rapid scientific acceptance of the principle of energy conservation to the analogy between energy and matter [36]. Feynman explains energy conservation in terms of counting a child's blocks [22]. diSessa, describing momentum, energy, charm, and other conserved field quantities states,

"These are all matter equivalents in the sense that they are conserved things whose distribution and flow in time and space are critical parameters of describing interactions. Experts, even in the most exotic corners of the field, reuse with a vengeance many reasoning patterns learned for matter. Are physicists guilty of overuse of ideas, such as conservation, originally learned in the context of matter? No. Should we perhaps applaud students for trying to extend matter-relevant descriptions to evidently nonmatter quantities, from "oomph" to "energy"? I would say, yes, and argue that, without the elaborated matter ontological class, learning physics of any sort would be impossible." [31]

Both experts and novices use multiple and overlapping metaphors for physics quantities that complement one another in complex representations of physical phenomena $[19,31,37]$. Our observations regarding the substance, stimulus, and vertical location metaphors for energy add to the evidence supporting this "dynamic ontologies" perspective in that they document variability in novices' and experts' ontologies for energy. These observations contradict earlier assertions that the substance and process ontologies are cognitively mutually exclusive, and that this "ontological distinctness" makes it "not possible to refine or develop intuitive knowledge to the point that it becomes the veridical physics knowledge" [11]. A dynamic model of novice and expert ontologies in physics suggests instruction that builds expert concepts from students' everyday resources and reinforces the beginnings of expertlike thinking skills as they appear in classroom discourse [38-40]. Our instructional approach is to identify a particular conceptual metaphor for energy - the substance metaphor-that is both intuitively accessible for students and conceptually productive and harness the specific affordances of that metaphor to make progress toward particular learning goals.

\section{B. Concerns about a substance ontology for energy}

Concerns about the use of the substance metaphor for energy in instruction center on the idea that energy is a purely mathematical quantity. This concern has been well articulated in several analyses [24,41-43]. Feynman states that energy is "not a description of a mechanism, or anything concrete" [22]. Arons, similarly, says,

"Energy is not a substance, fluid, paint, or fuel which is smeared on bodies and rubbed off from one to another. We use this term to denote a constructnumbers, calculated a certain prescribed way, that are found by theory and experiment to preserve a remarkably simple relationship in very diverse physical phenomena." [44] (emphasis in original)

Duit [44] dates this objection to the 19th century, in which Helm stated,

"The manifestations of energy belong to the world of the senses, but energy itself remains above these in the same way as the Platonic idea remains above objects. The energy concept is welcomed by its keenest proponents as doing justice to the facts and yet 
remaining so far above them that it excludes the danger of renewed substantivation." [45]

Our position is that the activity of invoking a metaphor and implicating its ontology is an essentially utilitarian act. The ontology inherent within a metaphor is used as a cognitive lever in advancing conceptual development; its use as such need not refer to the "true nature" of energy, nor need it assume that a unique true nature exists. To use the metaphor to organize thought is not to make a claim about the physical world or a transcendent reality of Platonic forms. In our view, a metaphor is not invalidated in an ultimate sense if it is shown to be untenable in some cases. Rather, those cases show only the limit of the metaphor's cognitive utility. For example, material substances have a number of properties, including being pushable, frictional, consumable, inertial, and gravity sensitive [35]; these are qualities which should not to be attributed to energy in introductory physics. On the other hand, other features of substances productively characterize energy in introductory physics, including being containable, localized, movable, additive, and conserved. Our use of the substance metaphor in instruction depends on learners attributing only some of the properties of material substances to energy. In other words, energy should be conceptualized as a quasimaterial substance [24]. The fact that the substance conceptualization of energy has limitations need not prevent us from using it to organize our understanding.

A different potential concern about a substance metaphor for energy is that it constitutes a rebranding of caloric theory, in which heat was modeled as a substance (caloric) that flowed from a body at high temperature (full of caloric) to a body at low temperature (with less caloric). In the modern theory of energy, heat is not a flowing substance; rather, it is a mechanical process of collisions, the result of which is to lower energy in one object and raise it in another. Thus, to model energy as a substance may suggest an outdated conceptualization of heat. From a different perspective, it may serve as the foundation for understanding both energy and momentum flow in advanced mechanics.

The description of energy as being located in objects can be a concern for gravitational and other forms of potential energy, which are properly located in a system of objects or in a field, rather than in individual objects. In our instructional contexts, we allow learners to locate such forms of energy either in isolated objects, in systems of objects, or in fields, as is appropriate to the level and learning goals of the course. This approach allows for iterative negotiation and refinement of the meaning of systems, an important learning goal in the study of energy. Alternatively, learners may identify a field as a new kind of (nonmaterial) object that can contain energy.

Other objections to the substance metaphor include the fact that energy is a frame-dependent and often negative quantity, properties not well represented by a substance conceptualization. The frame dependence of energy is an instructional refinement that, in and of itself, raises no insuperable objection to the substance metaphor, given that in any particular frame the substance metaphor is applicable and productive. The sign of energy requires a different refinement, namely, the realization that potential energy depends not only on the system of mutually interacting objects but also on a reference point.

In quantum mechanics, energy is not associated with a region of space, and one of the key features of the substance conceptualization of energy is lost [24]. Quantum mechanics presents such a drastic reconceptualization of object permanence that there is no surprise that energy would need to be reconceptualized as well.

\section{Summary}

In summary, instructional use of a substance metaphor for energy has limitations. The imagined substance has unusual properties: it is invisible, it is massless, it permeates objects, it occurs in different forms, and it transfers and transforms in the course of physical processes. The utility of the metaphor is mostly limited to classical, nonrelativistic situations within any fixed frame of reference. Metaphysical commitments about the "true nature" of energy may preclude any intentional use of the metaphor for some physicists, even for pedagogical purposes.

Our position is that within the limits described above, a substance metaphor for energy has advantages for physics understanding that outweigh the concerns, particularly for understanding energy conservation and transfer. In line with Amin [17], we understand the concerns about the substance conceptualization of energy to be resolved by the theory of conceptual metaphor, which characterizes the nature of the scientific concept of energy in such a way as to demonstrate the continuity between the naive and scientific concepts of energy. Evidence suggests that learners are able to navigate the subtleties involved in thinking of energy as a quasimaterial substance rather than a material substance $[17,23]$. We attribute this unproblematic use of the substance metaphor to learners' explicit understanding that it is a metaphor, not a description of physical features of energy as being equivalent to matter.

\section{GOALS FOR ENERGY LEARNING}

This research takes place as part of The Energy Project, a five-year NSF-funded project whose goal is to promote elementary and secondary teachers' development of formative assessment practices in the context of energy. Our goals for energy learning are specific to our population of learners and include conceptual understanding, sociopolitical relevance, creative flexibility, and representational competence. The focus of this paper is on the affordances of specific energy ontologies for conceptual understanding of energy. 
Among the primary needs of elementary and secondary teachers is foundational conceptual understanding of physics content [46-48]. Our conceptual learning goal is for learners to conserve energy as they track the transfers and transformations of energy within, into, or out of the system of interest in complex physical processes. National standards for energy learning identify conservation and tracking as high priorities, e.g., "Students should trace where energy comes from and goes next in examples that involve several different forms of energy along the way" [49]. Similar recommendations appear in Ref. [50]. Current research in the teaching and learning of energy in biology posits tracking as the marker of high achievement in energy learning progressions [51]. These learning goals are distinct from approaches that posit energy as primarily a numerical quantity and conceptualize energy conservation in terms of mathematical balancing.

\section{ONTOLOGIES FOR ENERGY IN GRAPHICAL REPRESENTATIONS}

Learning is structured by representations. We analyze classic representations of energy-bar charts, pie charts, and others-to determine the energy ontologies that are implicit in those representations, and thus their affordances for the energy learning goals relevant to our learner population. As we stated earlier, our guiding questions include:

- What do specific representations "say" about energy? In particular, what ontology of energy does each one articulate?

- What questions do specific representations "ask" of one who engages with them?

- Which representations best support our learning goals? In particular, which representations support conservation and tracking of energy in complex physical processes?

We find that while existing representations partially support a substance metaphor for energy and thus the learning goal of energy conservation, they have limited utility for tracking the flow of energy among objects.

\section{A. Bar charts}

A bar chart is a chart in which data values are represented with rectangles. Different rectangles indicate different categories of data, and the height (or length) of a rectangle indicates the quantity of data in that category. In energy bar charts, the categories are normally forms of energy (e.g., kinetic, potential); the height of each bar tells the amount of energy that has that form. Some energy bar charts also have a bar indicating the total amount of energy in the represented system; the heights of all the other bars must then sum to the height of the total-energy bar [52]. Energy conservation is indicated by the total-energy bar having constant height during processes that change the heights of the other bars. Animated bar charts, in which individual forms of energy change in amount while the total energy remains the same, emphasize energy conservation more strongly than static bar charts. A particular advantage of bar charts is that they can represent negative energies, which is difficult or impossible in other representations [23].

As a representational medium, bar charts primarily "say" that there are different categories of energy, thus supporting understanding of forms and transformation of energy. The main task of a user who engages with a bar chart is to determine the relative heights of the bars, i.e., the relative amounts of energy that are in different forms.

Bar charts are consistent with the substance metaphor in that they represent energy as being a kind of stuff; this stuff comes in stacks, like towers of books. However, in the bar chart representation energy is not "in" objects; rather it is "in" forms, or systems of objects. Bar charts do not entirely support the substance metaphor as articulated above, in which energy is "in" objects. Thus, though they can support energy conservation, they do not explicitly support the local tracking of energy transfers and transformations among objects.

\section{B. Pie charts}

A pie chart is a circular chart divided into sectors; the size of the sector is proportional to the quantity it represents. Pie charts are generally similar to bar charts in that they indicate the relative amounts of data that are in distinct categories, which are again forms of energy. However, a pie chart communicates distinctly that there is a total amount of energy (the whole "pie"), which is divided into parts (forms). The size of each sector tells the proportion of energy that is in that form. Energy conservation within a system is indicated by a constant-size pie under transformations of its component pieces. Energy transfers into or out of a system can be managed by increasing or decreasing the size of the pie [23].

Pie charts emphasize the unitary nature of energy $[23,32]$. They are consistent with the substance metaphor insofar as they represent energy as being a kind of stuff, in particular, a culturally familiar form of food. However, as in bar charts, the energy is not "in" objects, but "in" forms and systems of objects. Though proponents of pie charts claim that they support conservation, storage, and transfer [23], we claim that they must do so indirectly, since energy is not represented as being located in or moving among objects.

\section{PET diagrams}

The Physics and Everyday Thinking (PET) curriculum [53] is designed for prospective or practicing elementary teachers and is adapted from multiple projects grounded in research on student learning of physics [54,55]. The curriculum emphasizes energy descriptions of interactions and uses a distinctive representation in doing so. Though this representation is not widely used at the university level, it 
Contact Interaction

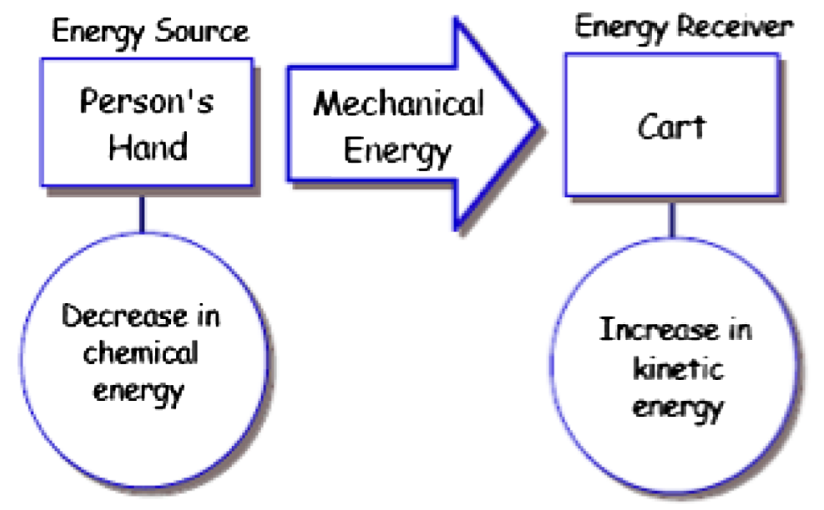

FIG. 1 (color online). Physics and Everyday Thinking (PET) energy diagram for a hand pushing a cart.

is well known in K-12 contexts, and initially seemed especially promising to us for use with a substance metaphor for energy.

In a PET energy diagram, objects are represented as rectangles; the name of each object is in the rectangle (see Fig. 1). Objects most often interact in pairs. The interaction is represented as involving an energy transfer from one interacting object (the "energy source") to the other (the "energy receiver"). The energy transfer is represented by means of an arrow pointing from the source to the receiver. Changes in the energy of the interacting objects are represented by means of circles attached to the objects from below; each circle contains a phrase such as "decrease in chemical energy" or "increase in kinetic energy."

The source-receiver layout of PET diagrams articulates a flow of energy from one object to another, in line with our learning goals (and better aligned in this sense than bar charts and pie charts). Forms appear in PET diagrams as labels for energy. While our own articulation of the quasimaterial substance metaphor does not specify the ontological status of forms, making it difficult to identify alignments with regard to this representational feature, the metaphor of forms as labels is at least not in conflict with our model. (In contrast, bar and pie charts represent forms as containers for energy, which conflicts with thinking of energy as being contained in objects.) The task of learners in completing a PET diagram is to answer questions such as, What is the source and what is the destination of the energy? What form of energy decreases in the source, and what form increases in the receiver? These questions align very well with what we expect of participants in our courses, and in the first year of the Energy Project, we strongly emphasized the use and interpretation of PET energy diagrams.

In practice, however, we have found that the PET energy diagram does not articulate a substance metaphor explicitly enough to support our learners in modeling energy dynamics. Because energy is not pictured explicitly in a PET diagram, there is ambiguity in localizing and tracking it. In
Fig. 1, for example, where is the energy? It is moving from the hand to the cart, but nothing is visibly lessened in the hand or increased in the cart. When we use this representation in instruction, we observe that some learners think of the energy as being in the boxes; some think of it as being in the circles, or being the circles; and some think of it as being the arrow. Others think the arrow represents time, in which case it is not clear whether the diagram represents the objects during the interaction, or both before and after it. Others think of the arrow as representing the mechanism of transfer-not energy, but how energy goes from the source to the receiver (perhaps a force). These ambiguities may contribute to interpretations such as Fig. 2, drawn by an elementary teacher in the first year of the Energy Project to represent a $\log$ burning to cook a hamburger. In this diagram, thermal energy is represented as being between the $\log$ and the hamburger, rather than in either object.

Another difficulty we have observed with PET energy diagrams is that the term "energy source" is misunderstood to refer to a supplier of energy that humans can use; learners want to put things like "battery," "solar," and "log" into the "source" box. We find that changing the name of the box from "source" to "giver" helps enculturate learners to the idea that in a physics context, any object can possess and transfer energy. Learners build on this idea by creating chains of PET diagrams that show a series of energy transfers, or branched diagrams that show energy transferring to more than one object at a time. The labels for forms of energy grow increasingly complex in these "PET-plus" diagrams, as learners strive to include multiple forms of energy that may be increasing, decreasing, or constant in each represented object.

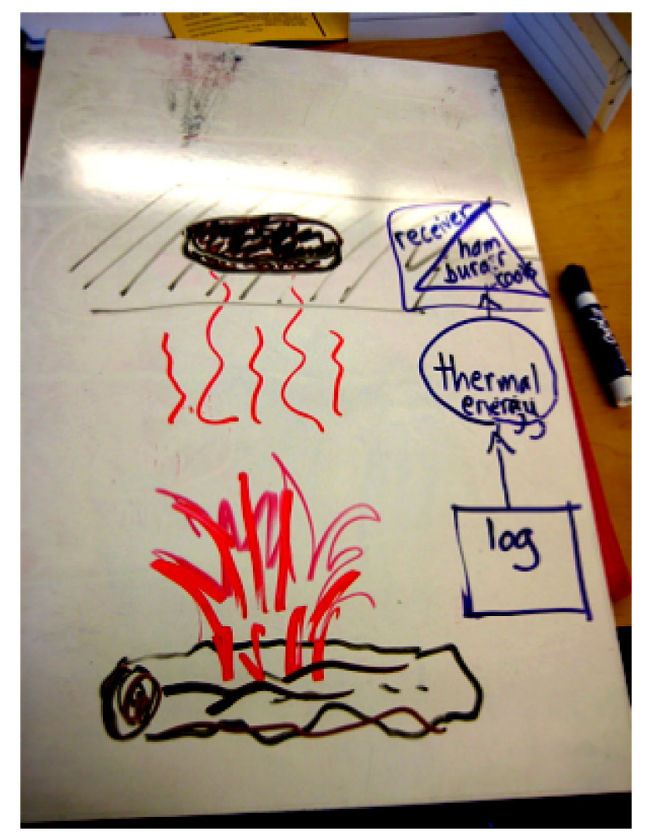

FIG. 2 (color online). Learner-generated diagram for a hamburger cooking over a fire, inspired by PET energy diagrams. 


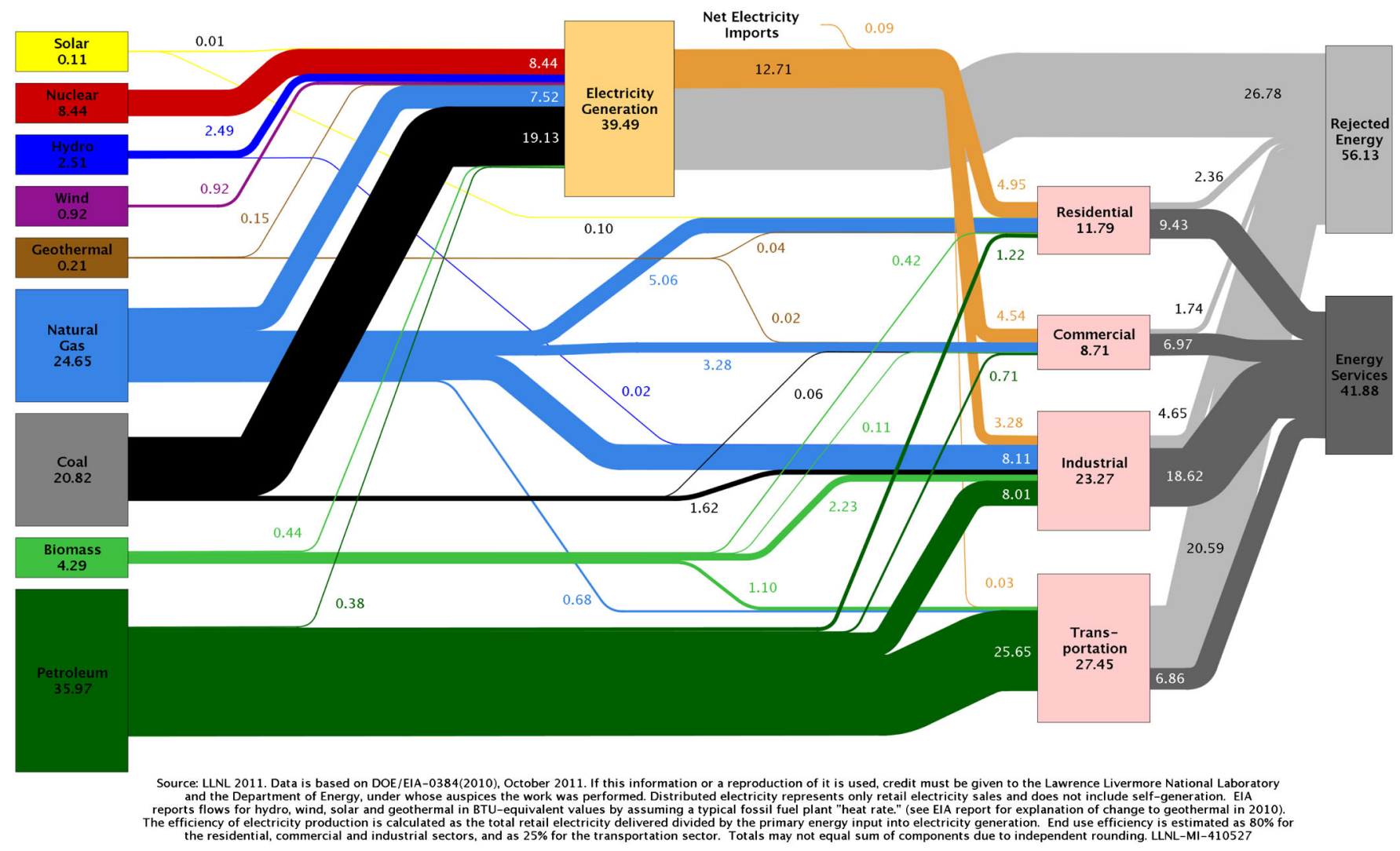

FIG. 3 (color online). Sankey energy diagram of energy use in the United States in 2010.

\section{Sankey diagrams}

A Sankey diagram is a flow diagram in which flow of a quantity is represented with arrows. Flow is shown as proceeding from a set of sources (typically arrayed in a column on the left) to a set of destinations (in a column on the right). The amount of flow is represented by the width of the arrow. In other words, energy is represented as being in arrows between objects (rather than in objects). Portions of the flow may branch and recombine in the middle of the diagram, representing intermediate processes or categorizations. Figure 3 is a Sankey diagram of energy use in the United States in 2010 [56]. Though Sankey diagrams are difficult to construct by hand, they are common in sociopolitical discussions of energy sources and usage.

Sankey diagrams combine some of the advantages of bar charts, pie charts, and PET diagrams: They show energy as coming from certain entities and going to others, thus supporting conservation and tracking, and they display the relative proportions of energy in different categories. To create a Sankey energy diagram, learners identify the "givers" and "receivers" of energy and also the relative proportions of the categories of energy that are given and received in each case. In Fig. 3, the givers (or sources) are means of supplying energy to human infrastructure, such as natural gas, coal, and petroleum, and the receivers (or users) are the infrastructure categories that require energy to function, such as residential and transportation. An option for introductory physics instruction would be to identify objects as givers and receivers, as in a PET diagram, but with variable-width arrows [32]. In Fig. 3, arrows mostly retain the color of their source, suggesting that the arrows represent energy that was generated by a certain means. This categorization is different than in introductory physics, where it is more typical to categorize energy by its form than by its source.

\section{E. Support for learning goals}

In summary, each of the aforementioned representations partially supports the learning goals of energy conservation and tracking. They all represent energy to some degree as being conserved, localized, and changing form. Bar charts and pie charts show energy as being in forms or systems of objects, rather than in individual objects, which limits their utility for tracking the flow of energy among objects.

An important limitation of all of the above representations is that both learners and instructors find themselves wishing for a dynamic representation-one that can show energy accumulating in objects, or represent the complexity of energy transfers and transformations as physical processes unfold. While it is possible to remedy this limitation 
with computer animations, generating such animations requires more technical skill and time than many learners (or instructors) have available, especially during a lesson.

\section{SUMMARY}

An embodied cognition theoretical perspective on the understanding of energy supports us in identifying three main ontologies for energy in learner and expert physics discourse: (1) a quasimaterial substance, (2) a stimulus to action, and (3) a vertical location. We identify a substance ontology for energy as having special advantages for physics instruction in which the emphasis is on conceptual understanding of local energy conservation, transfer, and flow. Though we are aware of concerns about the substance conceptualization of energy, the limitations that these concerns legitimately impose are acceptable for our particular instructional context, and do not outweigh the considerable advantages. Familiar graphical representations of energy - bar charts, pie charts, and others-do not fully support energy conservation and tracking in complex physical scenarios.
In a companion paper [5], we present novel representations that embody the substance metaphor and support learners in conserving and tracking energy as it flows from object to object and changes form. Such representations enable detailed modeling of energy dynamics in complex physical processes.

\section{ACKNOWLEDGMENTS}

We gratefully acknowledge all the elementary and secondary teachers who have participated in Energy Project courses for their generosity in making their own and their students' reasoning accessible to the Energy Project team. We are indebted to the members of the Physics Education Research Group at Seattle Pacific University, especially Eleanor W. Close, Lezlie S. DeWater, and Lane Seeley, for thoughtful discussions of this work. We also thank the national community of Energy Project collaborators for their engagement with our research, particularly Leslie J. Atkins, Michael C. Wittmann, Benedikt Harrer, and Jim Minstrell. This material is based upon work supported by the National Science Foundation under Grant No. 0822342.
[1] B. C. van Fraassen, Scientific representation: Paradoxes of perspective, Analysis 70, 511 (2010).

[2] B. Latour, Reassembling the Social: An Introduction to Actor-Network Theory (Oxford University Press, New York, 2005).

[3] E. Hutchins, How a cockpit remembers its speeds, Cogn. Sci. 19, 265 (1995).

[4] S. Sismondo, An Introduction to Science and Technology Studies (Blackwell Publishing, Malden, MA, 2006).

[5] R. E. Scherr et al., Representing energy. II. Energy tracking representations, following paper, Phys. Rev. ST Phys. Educ. Res. 8, 020115 (2012).

[6] R. E. Scherr, H. G. Close, and S. B. McKagan, Intuitive ontologies for energy in physics, AIP Conf. Proc. 1413, 343 (2012).

[7] G. Lakoff and M. Johnson, Philosophy in the Flesh: The Embodied Mind and Its Challenge to Western Thought (Basic Books, New York, 1999).

[8] G. Lakoff and R.E. Nuñez, Where Mathematics Comes From: How the Embodied Mind Brings Mathematics into Being (Basic Books, New York, 2000).

[9] M. T. H. Chi and J. D. Slotta, The ontological coherence of intuitive physics, Cogn. Instr. 10, 249 (1993).

[10] M. T. H. Chi, Commonsense conceptions of emergent processes: Why some misconceptions are robust, J. Learn. Sci. 14, 161 (2005).

[11] J. D. Slotta and M. T. H. Chi, Helping students understand challenging topics in science through ontology training, Cogn. Instr. 24, 261 (2006).

[12] D. M. Watts, Some alternative views of energy, Phys. Educ. 18, 213 (1983).
[13] G. Nicholls and J. Ogborn, Dimensions of children's conceptions of energy, Int. J. Sci. Educ. 15, 73 (1993).

[14] R. Trumper, A cross-age study, Int. J. Sci. Educ. 15, 139 (1993).

[15] J. Bliss and J. Ogborn, Children's choices of uses of energy, Eur. J. Sci. Educ. 7, 195 (1985).

[16] J. Solomon, Learning about energy: How pupils think in two domains, Eur. J. Sci. Educ. 5, 49 (1983).

[17] T.G. Amin, Conceptual metaphor meets conceptual change, Hum. Dev. 52, 165 (2009).

[18] J. D. Slotta, M. T. H. Chi, and E. Joram, Assessing students' misclassifications of physics concepts: An ontological basis for conceptual change, Cogn. Instr. 13, 373 (1995).

[19] A. Gupta, D. Hammer, and E. F. Redish, The case for dynamic models of learners' ontologies in physics, J. Learn. Sci. 19, 285 (2010).

[20] R. A. Serway and J. W. Jewett, Physics for Scientists and Engineers with Modern Physics (Thomson Higher Education, Belmont, CA, 2007), 7th ed.

[21] D. Halliday, R. Resnick, and J. Walker, Fundamentals of Physics (John Wiley \& Sons, Hoboken, NJ, 2008).

[22] R. P. Feynman, R. B. Leighton, and M. Sands, The Feynman Lectures on Physics (Addison-Wesley, Reading, MA, 1969).

[23] E. Brewe, Energy as a substancelike quantity that flows: Theoretical considerations and pedagogical consequences, Phys. Rev. ST Phys. Educ. Res. 7, 020106 (2011).

[24] R. Duit, Should energy be illustrated as something quasimaterial?, Int. J. Sci. Educ. 9, 139 (1987).

[25] G. Falk, F. Herrmann, and G. Bruno Schmid, Energy forms or energy carriers?, Am. J. Phys. 51, 1074 (1983). 
[26] M. C. Wittmann, J. T. Morgan, and L. Bao, Addressing student models of energy loss in quantum tunneling, Eur. J. Phys. 26, 939 (2005).

[27] D. Domert, C. Linder, and A. Ingerman, Probability as a conceptual hurdle to understanding one-dimensional quantum scattering and tunneling, Eur. J. Phys. 26, 47 (2005).

[28] S. B. McKagan and C.E. Weiman, Exploring student understanding of energy through the quantum mechanics conceptual survey, in Proceedings of the Physics Education Research Conference, Salt Lake City, Utah, 2005, edited by P. R. L. Heron, L. McCullough, and J. Marx (AIP, New York, 2005).

[29] S. B. McKagan, K. K. Perkins, and C. E. Wieman, Deeper look at student learning of quantum mechanics: The case of tunneling, Phys. Rev. ST Phys. Educ. Res. 4, 020103 (2008).

[30] D. T. Brookes and E. Etkina, Do our words really matter? Case studies from quantum mechanics, in Proceedings of the Physics Education Research Conference, Salt Lake City, Utah, 2005 (Ref. [28]).

[31] A. diSessa, Ontologies in pieces: Response to Chi and Slotta, Cogn. Instr. 10, 272 (1993).

[32] G. Swackhamer, Cognitive resources for understanding energy, http://modeling.asu.edu/modeling/ CognitiveResources-Energy.pdf.

[33] R. Millar, "Teaching about energy," Department of Educational Studies Research Paper, York University, 2005).

[34] S. B. McKagan et al., Criteria for creating and categorizing forms of energy, AIP Conf. Proc. 1413, 279 (2012).

[35] M. Reiner, J. D. Slotta, M. T. H. Chi, and L. B. Resnick, Naive physics reasoning: A commitment to substancebased conceptions, Cogn. Instr. 18, 1 (2000).

[36] M. Planck, Das Prinzip von der Erhaltung der Energie (B. G. Teubner, Leipzig, 1913).

[37] D. T. Brookes and E. Etkina, Using conceptual metaphor and functional grammar to explore how language used in physics affects student learning, Phys. Rev. ST Phys. Educ. Res. 3, 010105 (2007).

[38] D. Hammer and E. van Zee, Seeing the Science in Children's Thinking: Case Studies of Student Inquiry in Physical Science (Heinemann, Portsmouth, NH, 2006).

[39] D. Hammer, Student resources for learning introductory physics, Am. J. Phys. 68, S52 (2000).
[40] L. J. Atkins and I. Y. Salter, Constructing definitions as a goal of inquiry, AIP Conf. Proc. 1289, 65 (2010).

[41] P. Lijnse, Energy between the life-world of pupils and the world of physics, Sci. Educ. 74, 571 (1990).

[42] J. Warren, The nature of energy, Eur. J. Sci. Educ. 4, 295 (1982).

[43] J. Warren, At what stage should energy be taught?, Phys. Educ. 21, 154 (1986).

[44] A. B. Arons, Development of Concepts of Physics: From the Rationalization of Mechanics to the First Theory of Atomic Structure (Addison-Wesley, Reading, MA, 1965).

[45] G. Helm, Die Lehre von der Energie (A. Felix, Leipzig, 1887).

[46] L.C. McDermott and L.S. DeWater, The need for special science courses for teachers: Two perspectives, in Inquiring into Inquiry in Science Learning and Teaching, edited by J. Minstrell and E. van Zee (AAAS, Washington, DC, 2000).

[47] P.S. Shaffer and L. C. McDermott, A research-based approach to improving student understanding of kinematical concepts, Am. J. Phys. 73, 921 (2005).

[48] L. C. McDermott, P.S. Shaffer, and C.P. Constantinou, Preparing teachers to teach physics and physical science by inquiry, Phys. Educ. 35, 411 (2000).

[49] American Academy for the Advancement of Science, Benchmarks for Science Literacy (Oxford University Press, New York, 1993).

[50] A Framework for K-12 Science Education: Practices, Crosscutting Concepts, and Core Ideas (National Academies Press, Washington, DC, 20112).

[51] H. Jin and C.W. Anderson, Learning progression for energy in carbon-transforming processes (unpublished).

[52] A. van Heuvelen and X. Zou, Multiple representations of work-energy processes, Am. J. Phys. 69, 184 (2001).

[53] F. Goldberg, V. Otero, and S. Robinson, Physics and Everyday Thinking (It's about time, Inc., Armonk, NY, 2008).

[54] F. Goldberg et al., Constructing Ideas in Physical Science: Field Test Version (It's About Time, Armonk, NY, 2003).

[55] F. Goldberg, V. Otero, and S. Robinson, Design principles for effective physics instruction: A case from Physics and Everyday Thinking, Am. J. Phys. 78, 1265 (2010).

[56] Lawrence Livermore National Laboratory and Department of Energy, Estimated U.S. Energy Use in 2010, 2012 [cited 2012 April 14], available from https://flowcharts.llnl.gov/content/energy/energy_archive/ energy_flow_2010/LLNLUSEnergy2010.png. 